\title{
Análisis espacial del sitio arqueológico San Pedro, ubicado en el bajo río San Jorge, Caribe colombiano
}

\author{
Spatial Analysis of Archaeological Site of San Pedro Located \\ in Lower San Jorge River, Colombian Caribbean
}

\begin{abstract}
Sneider Rojas Mora
Laboratorio de Arqueología, Departamento de Antropología, Universidad de Antioquia, Colombia sneider.rojas@udea.edu.co
\end{abstract}

Fernando Montejo Gaitán

Instituto Colombiano de Arqueología e Historia, Colombia

fmontejo@icanh.gov.co

\begin{abstract}
$\overline{\text { RESUMEN }}$
El sitio arqueológico de San Pedro, ubicado en el área de influencia del caño Rabón en la Depresión Momoposina, es uno de los lugares mejor preservados de la región. Identificado por Plazas y Falchetti en la década del setenta del siglo pasado, constituye un complejo sistema de canales y camellones que circundan una extensa zona de plataformas ubicadas a la manera de un poblado central. En la parte externa a los canales y camellones, también se identifican grandes conjuntos de plataformas, en este caso con montículos que parecen corresponderse con montículos funerarios. Este sitio, datado en el año 680 ( \pm 120$)$ d. C., hace parte del registro de los primeros siglos de ocupación de la región. En el presente artículo se hace un análisis espacial de dicho conjunto arquitectónico y se establecen las diferencias entre los elementos que lo conforman.
\end{abstract}

Palabras clave: campos de cultivo, sistema hidráulico prehispánico, Caribe colombiano, Depresión Momposina.

\begin{abstract}
$\overline{\text { ABSTRACT }}$
The archaeological site San Pedro, located in the area of influence of Rabón stream in Depresión Momposina is one of the best preserved places of the region. Plazas and Falchetti identified during the nineteen seventies, a complex system of channels and ridges that surround a large area of platforms located towards a central village. On the outside of channels and ridges, large sets of platforms are also identified, this time with artificial hills that seem to correspond with burial mounds. This site dated $680( \pm 120) A D$, is part the first evidence of occupation in the region. In this article, we discuss the spatial analysis of the architectural setting of these sites, and the differences among them.
\end{abstract}




\section{Introducción'}

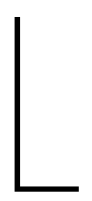

a transformación del entorno mediante la construcción de cualquier tipo de estructura deja vestigios en el espacio que dan cuenta de las construcciones sociales que hombres y mujeres hicieron allí en un determinado tiempo. Ejemplo de ello son las estructuras físicas presentes en zonas pantanosas, como canales, camellones y plataformas, que han quedado como testigos de las adecuaciones, modificaciones, vivencias y usos de dichos ambientes por parte de las sociedades que los ocuparon. No obstante, un uso o una vivencia no siempre dejan un único tipo de vestigio y, por lo tanto, no siempre se puede establecer una correspondencia directa entre tipos de vestigios y de vivencias, usos o fines; es decir que difícilmente se puede recurrir a las analogías simples, como aquellas que indican que una forma se corresponde con una función y que esa forma y esa función, sumadas a las dimensiones, proporcionan una información confiable respecto al tipo de sociedad que se observa. Se olvida con ello que las formas pueden responder a prácticas de transformación distintas y, por consiguiente, a estructuras sociales diversas, y que las dimensiones pueden estar asociadas, entre otros factores, al tiempo y al entorno mismo.

En casi todas las sociedades se producen modificaciones del entorno debidas al desgaste, el transporte y la acumulación de suelos y material parental, lo que hace evidente que, para adentrarse en cualquiera de los sistemas de la estructura social de quienes los produjeron, es necesario organizar conjuntos definidos bien sea por la forma (plataformas, montículos, camellones, aterrazamientos) o por las funciones (vivienda, agricultura, espacio público, etc.). Pero se debe hacer la salvedad de que dichas taxonomías responden a interrogantes y estructuras mentales de quien investiga, y que para conocer su relación en el pasado es preciso tener en cuenta los procesos históricos que siguieron las sociedades que las produjeron.

El presente artículo es resultado parcial de un proyecto de investigación inscrito en el programa de investigación en arqueología "Trayectorias prehispánicas de complejización social en la Depresión Momposina". Este programa fue diseñado en la Universidad de Antioquia y el ICANH, y de él hacen parte varios proyectos de investigación adelantados por el profesor Sneider Rojas Mora, los cuales han sido financiados por la Vicerrectoría de Investigación, la Vicerrectoría de Docencia y los Proyectos CODI de la Universidad de Antioquia. También se ha contado con el apoyo de sostenimiento a los grupos de investigación de la SIU de la misma universidad. De igual forma, es el resultado de los trabajos de investigación llevados a cabo por el Dr. Fernando Montejo Gaitán, financiados por el ICANH y por el Grupo de Investigación en Arqueología de la misma institución. 
En cuanto al fin agrícola, América es una de las regiones del mundo en las que se ha registrado el mayor número de zonas modificadas, en formas que van desde sencillos pozos para almacenar el agua hasta complejos sistemas de manejo hidráulico y canalización de ríos. Estas obras de adecuación, relacionadas con la mejora en la fertilidad de suelos para el cultivo intensivo o extensivo, y también con el drenaje y la irrigación, han sido la base sobre la cual se han construido hipótesis que ven en dichos sistemas una estrategia para el sostenimiento de densas poblaciones humanas (Denevan 1992, 2001; Denevan y Turner 1974), la presencia de complejos e intrincados sistemas burocráticos de administración o, también, estructuras políticas determinadas. No obstante la diversidad que implican las formas expuestas en el espacio, son escasas las investigaciones que superan el límite de la descripción formal del sistema o la morfología (canales largos, cortos, entrecruzados, etc.); o aquellas que definen su función general (vías de comunicación, drenaje e irrigación) o las particularidades de la finalidad principal o secundaria (fertilidad de suelos, plantas cultivadas, piscicultura, fuente de nutrientes para el suelo, etc.), y mucho más escasas aquellas investigaciones que se atreven a integrar la adecuación del paisaje a los sistemas sociales, bien como causa o como efecto del cambio o la continuidad de las estructuras sociales (Erickson 2006, 2008, 2010; Iriarte et al. 2010; Lombardo 2010; Lombardo et al. 2011, 2013; Rodrigues 2014; Rostain 2010; Walker 1999, 2000, 2004, 2011).

El presente documento es el resultado de la investigación llevada a cabo en el sitio conocido como San Pedro, ubicado en jurisdicción del municipio de San Benito Abad (departamento de Sucre), en área de influencia del caño Rabón dentro de la Depresión Momposina. Este sitio, reportado por Clemencia Plazas y Ana María Falchetti a finales de la década del setenta del siglo pasado, muestra una gran concentración de campos elevados y plataformas, que quizás sean los mejor preservados en la región. Durante el reconocimiento en superficie llevado a cabo en el año 2012 se identificaron tres zonas, a saber: 1) agrupación de plataformas de diversos tamaños, 2) zona de canales y camellones cruzados y 3) zona externa sin canales ni camellones, pero con gran concentración de plataformas sobre las que, de acuerdo con la información gráfica de Plazas et al. (1993), se presentan túmulos funerarios que no han sido verificados en campo. El objetivo de esta investigación fue brindar un análisis espacial que permitiese aportar a la cimentación de futuras investigaciones destinadas a estudiar asuntos propios de la organización social y del orden político prehispánico en la región. Para cumplir con este objetivo se utilizaron recursos técnicos especializados de sistemas de información geográfica.

En este artículo se asume que en la unidad geomorfológica denominada Depresión Momposina, en la que se identifica una gran modificación del paisaje, 
se dieron continuas construcciones de este que, en muchos casos, fueron similares entre sí, es decir, que existieron diferentes maneras de percibir el entorno inundable que se ofrecía. Somos conscientes de que el siguiente escrito es un ejercicio de investigación al que se le deben agregar elementos centrales, como la información cronológica. No obstante, se presenta como una hipótesis de trabajo apoyada en la información cartográfica de los trabajos de Plazas y Falchetti durante varias décadas de investigación, en los mapas digitalizados por Fernando Montejo y en el trabajo de campo llevado a cabo en el año 2012.

\section{La Depresión Momposina: el entorno}

Se denomina Depresión Momposina a una vasta llanura inundable ubicada en la parte central del Caribe colombiano, en jurisdicción administrativa de los departamentos de Córdoba, Sucre y Bolívar. En dicha unidad espacial vierten sus aguas los ríos Cauca, Magdalena, San Jorge y Cesar, con lo que se forman numerosas ciénagas permanentes y temporales, además de un extenso sistema de canales y caños que llevan y traen agua durante buena parte del año. La región está conformada por varias unidades geomorfológicas que le brindan sus características y la hacen particular en la costa caribe (figura 1). Al noroccidente de la Depresión hay una serie de colinas suaves formadas durante el Terciario; en el centro y surcado por fallas geológicamente activas, como El Colorado, Ayapel, Chicagua y Romeral, se ubica un plano denominado geomorfológicamente como llanura aluvial de desborde, que se remonta, igualmente, a un origen marino del Cretácico y sobre el cual se fue depositando material de origen continental. Entre tanto, la parte suroccidental con el mismo sustrato terciario tiene como característica geomorfológica la intensa participación del río Cauca, que forma un activo cono de deyección (Ballesteros 1983; Forero, Ferreira y Maya 1997; IGAC 1986). Adicionalmente, en la zona central de la Depresión, se presenta un continuo hundimiento, calculado por el Proyecto Colombo-Holandés entre 1,8 y 2,5 milímetros al año (Himat 1977a, 1977b), producto de las fallas antes mencionadas y del material continental agregado. De acuerdo con Van der Hammen (1986), por lo menos 40 o 50 metros de sedimentos de la Depresión Momposina tienen una edad máxima de 11.000 años antes del presente.

En esta región se presentan dos unidades geomorfológicas: 1) planicie fluvial constituida por una extensa región localizada entre los ríos San Jorge y Cauca, que, por su posición baja y su relieve plano, sufre inundaciones y 
Figura 1. Ubicación general de la Depresión Momposina en el Caribe colombiano

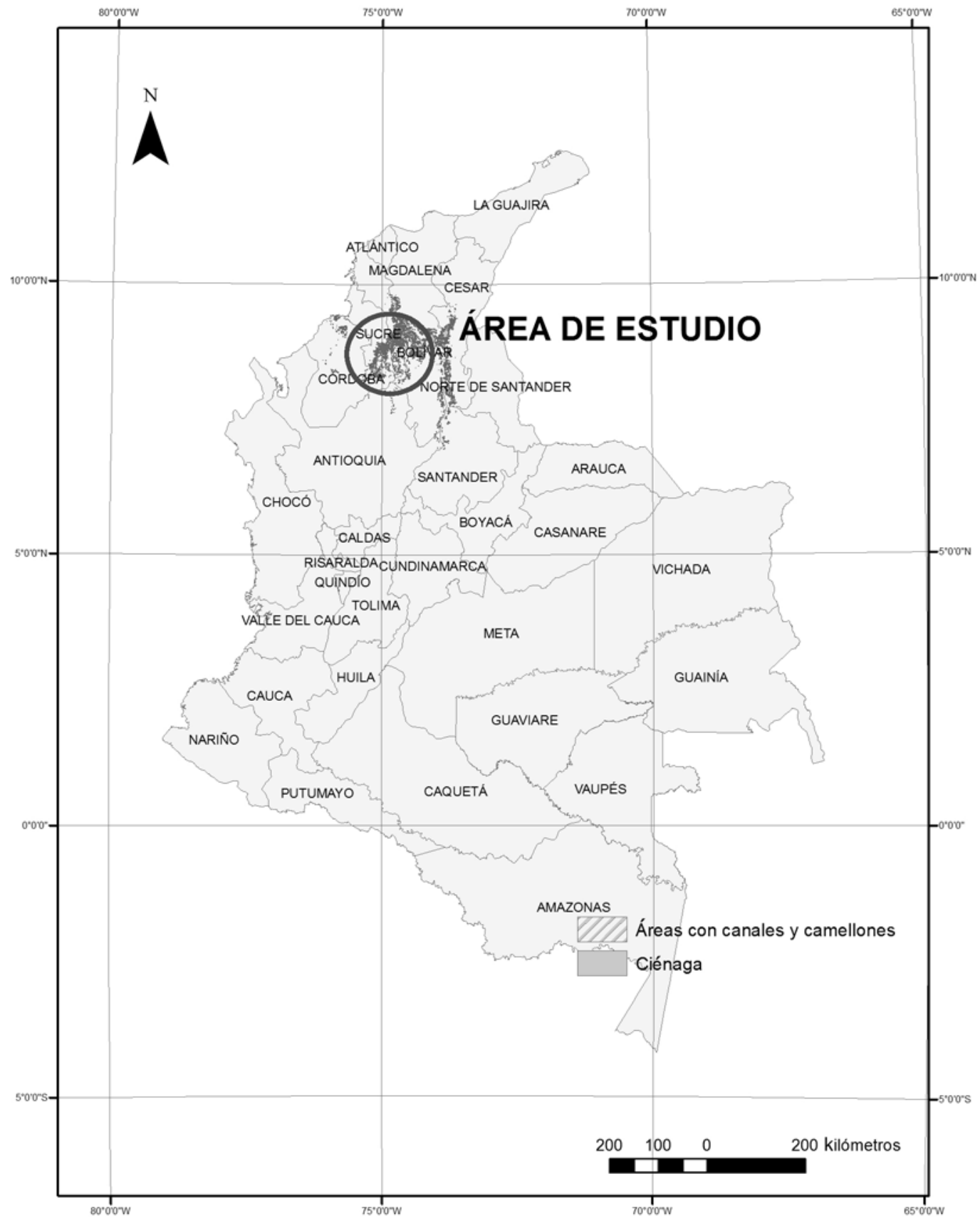

Fuente: Elaboración propia con base en imágenes de Google Earth. 
encharcamientos periódicos causados por desbordamientos; 2) planicie fluviolacustre, caracterizada por superficies planas y onduladas de origen fluvial y marino que descansan sobre un basamento del Terciario y en donde han actuado procesos erosivos, tanto geológicos como hídricos. De esta forma, los rebordes de ciénagas forman superficies estrechas, de relieve plano-cóncavo, que están localizadas en las orillas de las ciénagas y sufren inundaciones periódicas durante las épocas de invierno; hay acumulación de sedimentos heterométricos con predominio de materiales finos, que provienen de las terrazas y colinas adyacentes (IGAC 1986).

La abundante cantidad de sedimentos de diferentes tamaños que traen los cursos de agua se va depositando lentamente en sus lechos, lo que hace que finalmente sea menor la capacidad de carga de los ríos y se terminen desbordando sobre sus propios diques e inundando los basins. Estos últimos no siempre tienen manera de evacuar el agua de manera fluida y acaban formando ciénagas estacionales, que en algunos momentos se vuelven a cargar de sedimentos y buscan la salida rompiendo en astilleros los diques de los ríos y caños, lo que da pie a nuevos cursos de agua y, con ello, a nuevos diques naturales.

Entre los muchos caños permanentes cuyo curso pasa por la Depresión, podemos mencionar como principales aquellos ubicados en la región centrooccidental, como Rabón, Carate, Pajaral, San Matías, Los Ángeles, Vitoria y los caños Chicagua y Panzeguita en la región oriental. Así mismo, podrían señalarse muchos espejos de agua conocidos como ciénagas, que hacen las veces de amortiguadores durante la dinámica de aumento y descenso de los niveles de agua en la región.

De acuerdo con el registro histórico de las últimas décadas, el intervalo de mayor precipitación se presenta entre los meses de mayo y julio y entre los de octubre y noviembre. El total de precipitación anual es de $2.500 \mathrm{~mm}^{3}$ (IGAC 1986). La situación interna y externa anteriormente descrita hace que la Depresión Momposina permanezca inundada durante por lo menos ocho meses al año. Además, a esta condición de inundación se suma que la temperatura media anual en las tierras bajas del Caribe colombiano es de $26^{\circ} \mathrm{C}$, y la radiación solar de la región, de entre 2.600 y 3.100 calorías $/ \mathrm{cm}^{2}$ por semana, lo que indica una alta evaporación; esta condición actual, de cuya existencia en el pasado no tenemos conocimiento, es importante si consideramos que los cultivos requieren de un escenario arborizado que favorezca el equilibrio ecológico. En cuanto a la humedad relativa del aire, es decir, la relación entre el peso del vapor de agua del aire y el que podría contener si estuviese saturado a la temperatura ambiente del momento, su promedio anual es de $83,5 \%$, y los meses de mayo y junio son los 
que presentan los mayores indicadores (Agujera 2005). Estos hechos llevan a la constitución de suelos particulares, así como a la aparición de vegetación y fauna propias que aportan a la dinámica de la Depresión.

Los suelos de la región son de tipo aluvial y fluvioaluvial, cuya característica principal es la presencia de arcillas caolinitas, arenas y cuarzos que proveen información acerca de las condiciones climáticas y la transformación que han sufrido a lo largo de varios miles de años (Berrío et al. 2001). Adicionalmente, se ha considerado que la constante sedimentación, producto de la inundación, no permite la descomposición de la materia orgánica, lo que provoca ambientes con baja óxido-reducción, es decir, que no favorecen procesos de pedogénesis (IGAC 1986). La cantidad de material arcilloso, como las caolinitas, hace que haya terrenos poco drenados (figura 2).

En cuanto a la vegetación, el Himat (1977a, 1977b) hizo un detallado balance de la flora y distinguió cuatro tipos de vegetación: 1) bosque seco tropical (bs-T), en el que se encuentran especies como Attalea butyracea, Syagrus sancona, Acrocomia antioquensis excelsum, Amaranthus hybridus, Anacardium excelsum, Aspidospera, Bursera tomentosa, Capparis indica, Capparis odorata y Casearia corymbosa, así como Eichornia crasspes, Eleocharis interstincta, Hydrocotyle umbellata, Ludwigia pilosa, Pistia stratiotes, Polygonum densiflorum, Salvinia sprucei y Typha angustifolia; 2) bosque húmedo premontano (bh-PM), que se ubica principalmente en el valle del río Magdalena y cuyas especies más representativas son Clidemia capitellata, C. octona, Miconia aeruginosa, Miconia stenostachya, Clusia sp., Croton sp., Cupania sp., Cordia alliodora, Didymopanax morototoni, Erythrina poeppigiana, Ficus sp., Inga densiflora, Ladenbergia magnifolia, Nectandra sp., Ochroma lagopus, Myrsine guianensis, Trichanthera gigantea y Triumfetta mollissima; 3) bosque húmedo tropical (bh-T), que es un tipo de vegetación más frecuente en las riberas de los ríos: Acalypha macrostachya, Alchornea sp., Cassia reticulata, Cassia spectabilis, Calliandra sp., Erythrina edulis, Cecropia sp., Vismia sp., Piper aduncum, Tabebuia rosea, Tecoma mollis, Trema micrantha, Warszewiczia coccinea, Spondias mombin, Ilex sp., Tapirira guianensis y Virola sebifera; 4) vegetación de sabana, presente en momentos en que las condiciones climáticas lo favorecen, es decir, cuando se corresponden con una precipitación anual de $500 \mathrm{~mm}$. Esta última es una vegetación herbácea caracterizada por las familias Poaceae (Bouteloua filiformis, Cynodon dactylon), Cyperaceae (Cyperus feraz) y Asteraceae (Aspilla tenella). Los árboles de la vegetación de sabana solo se ven representados por Curatella americana y Byrsonima crassifolia. Es un ambiente vegetal en el que habitan varias especies animales representativas de la Depresión Momposina, algunas de las cuales están en vías de extinción debido a diferentes prácticas y 
Figura 2. Ubicación general de la Depresión Momposina

(el círculo indica el área de estudio, sitio de San Pedro)

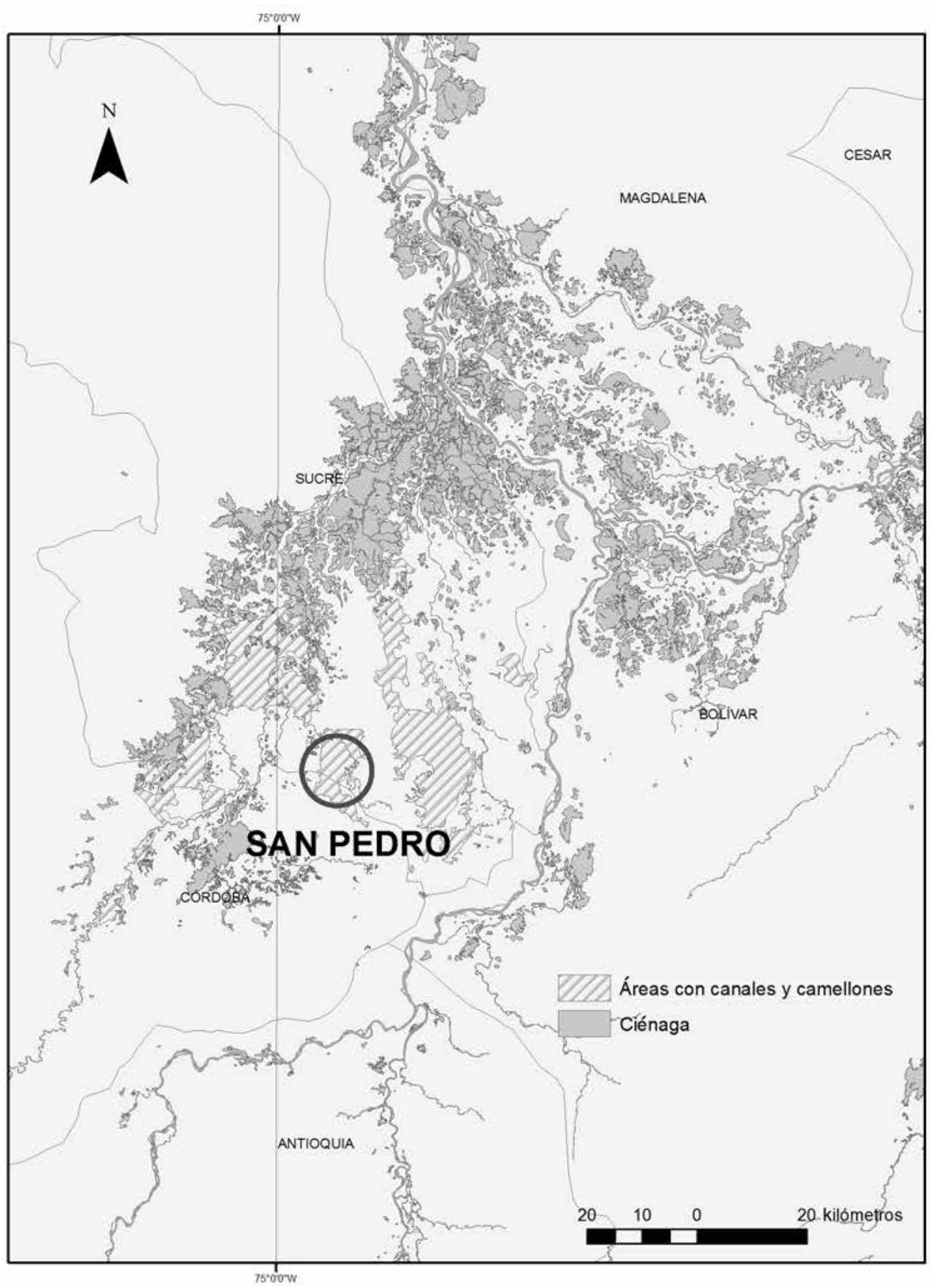

Fuente: Modificado por los autores a partir de Invías (1999). 
costumbres: a) peces como Prochilodus reticulatus magdalenae (bocachico), Pimelodus clarias (barbudo), Tarpon atlanticus (sábalo), Pseudoplatystoma fasciatum (bagre), Ageneiosus sp. (doncella), Leporinus muyscorum (comelones), Plecostomus tenuicauda (cacucho), Brycon moorei sinuensis (dorada), Hoplias malabaricus (moncholo), Brycon moorei (sardinata); b) aves como Dendrocygna autumnalis (pato pisingo), Tinamus major (gallineta), Platalea ajaja (cuchara), Casmerodius albus (garza de ganado), Ardea cocoi (garza morena), Brotogeris sp. (loro), Columba subnacea (tórtola); c) reptiles como Chelonoidis carbonaria (tortuga morrocoy), Trachemys callirosyris (tortuga hicotea), Iguana iguana (iguana), Cnemidophorus lemniscatus lemniscatus (lagartija), Caiman sclerops fuscus (babilla), Crocodylus acutus (caimán), Crotalus sp. (cascabel), Constrictor atrox (boa), Drymobius margaritiferus (azotadora); d) mamíferos como Felis pardalis (gato de monte), Didelphis marsupialis (chucha), Dasypus novemcinctus (ñeque, armadillo), Sylvilagus sp. (conejo), Agouti paca (guatinajo), Odocoileus virginianus (venado sabanero) y Mazamasp. (venado).

En este complejo ambiente lacustre se dio una apropiación y transformación del entorno, la construcción de nuevos paisajes (Herrera y Berrío 1996; Herrera, Rojas y Montejo 2004; Montejo y Rojas 2009; Plazas et al. 1988; Plazas y Falchetti 1981). Esta modificación se manifiesta en la presencia de estructuras de tierra que no se corresponden con diseños naturales, como camellones, canales, plataformas y montículos, en los que se observan, tras la excavación, diversas capas estratigráficas con características diferentes a las de los materiales adyacentes. Plazas y Falchetti mencionan que, "aunque a primera vista podrían parecer de origen natural, su contenido total de fosfato inorgánico (PO3/4) derivado de la actividad humana (302 y 275 ppm) corresponde a agricultura permanente razonablemente intensiva” $(1981,24)$.

En la actualidad, los camellones, plataformas y montículos tienen superficies erosionadas y son pocas las zonas que se conservan debido a procesos de ganadería itinerante en la región y a prácticas como la guaquería y el cultivo de arroz. En cuanto a los canales, estos se ven frecuentemente afectados por los procesos de sedimentación, producto de la dinámica fluvial y antrópica reciente.

Tal y como se mencionaba anteriormente, a esta zona llegan muchos materiales en suspensión que se depositan en los basins, en los que, casi siempre, se encuentran ubicados los distintos elementos del sistema prehispánico de camellones. Actualmente existe una nueva manera de relacionarse con el entorno, que también implica entender la dinámica de la región (inundación y materiales en suspensión), pero que establece esa relación de forma diferente; es decir que se abren canales y se rompen diques para llevar agua a los basins, con lo cual se 
logra una rápida sedimentación de los canales prehispánicos y la integración del terreno en una sola superficie plana, frecuentemente inundada y utilizada para la agricultura de arroz o la ganadería itinerante.

\section{La Depresión Momposina: contexto arqueológico}

El registro del sistema de canales y camellones de la Depresión Momposina inicia en la década del sesenta, cuando Parsons y Bowen (1966) advierten, a través de fotografías aéreas, la presencia de estructuras que consideraron antrópicas. No obstante, solo en los años setenta del siglo pasado se comienza a investigar de manera sistemática la región, mediante los trabajos de arqueología sistemática hechos por Clemencia Plazas y Ana María Falchetti, del Museo del Oro de Bogotá. Durante estas investigaciones se identificaron, mediante estudios aerofotogramétricos y algunas imágenes de radar, los principales sistemas de canales y camellones ubicados a lo largo de los caños Rabón y Mojana y el antiguo curso del río San Jorge (actuales caños La Pita, Mabobo, Carate, Pajaral y Los Ángeles) (Plazas et al. 1988, 1993; Plazas y Falchetti 1981).

También se hizo trabajo de campo en plataformas, túmulos funerarios y camellones, con el objetivo de obtener material arqueológico que permitiese establecer una cronología relativa de la región. En estas exploraciones, llevadas a cabo mediante cortes estratigráficos en túmulos funerarios y algunos pozos de sondeo en plataformas de vivienda, se obtuvieron y definieron tres conjuntos cerámicos (tradición granulosa incisa, tradición modelada pintada y tradición incisa alisada), que fueron asociados con desarrollos cerámicos de amplia dispersión en la costa atlántica (Plazas et al. 1993; Plazas y Falchetti 1981).

A lo largo de estas décadas de estudio, se consideró la región de influencia del caño Rabón como una de las de mayor potencial de investigación para entender las dinámicas de poblamiento de la región, en razón a los sitios tempranos con material arqueológico propio de la tradición granulosa incisa (p. ej. el sitio El Cogollo, siglo II a. C.) (Plazas et al. 1993). De acuerdo con Plazas et al. (1993), durante el periodo comprendido entre los siglos II a. C. y II d. C., en la misma área de influencia del caño Rabón, en el sitio de Marusa, hubo una transformación continua de la cerámica perteneciente a la tradición modelada pintada, que era tecnológica, estilística y funcionalmente distinta de la granulosa incisa, lo que 
llevó a estas autoras a sugerir un lento cambio cultural. Para ellas, este cambio, identificado con la tradición cerámica modelada pintada, no significó una sustitución sino un proceso de transformación continua relacionada con probables influencias foráneas (Plazas et al. 1993). Plazas y Falchetti (1981) mencionan que, en este sector, el "patrón de vivienda" fue lineal y disperso, tal como se observa en los caños Mabobo y Carate. En esta región no se han observado huertas domésticas aledañas a los asentamientos del eje principal. Sin embargo, existen extensas zonas de cultivo en la ciénaga de la Cruz o en San Pedro que, según Plazas et al. (1993), pertenecían a los habitantes asentados en las plataformas ubicadas a orillas del caño o en las plataformas localizadas dentro de los sistemas ajedrezados.

Las recientes investigaciones en la región dan cuenta de una ocupación datada en el año $680 \pm 120$ d. C. (Beta Analytic 2601); las muestras de carbón fueron obtenidas en un basurero que se encuentra en fase de análisis (Rojas 2015). El objetivo de dichas investigaciones es aportar al refinamiento de la cronología establecida para la región.

\section{Organización de datos espaciales}

El análisis espacial que se llevó a cabo en el sitio San Pedro siguió los protocolos propios de la investigación en sistemas de información geográfica. Inicialmente se utilizaron planchas aerofotográficas de la región de estudio a una escala de resolución de 1:25.000, así como planimetría de la región que había sido diseñada por el Museo del Oro a partir de fotografía aérea y sistematizada por Montejo y Ávila (2007), quienes además ampliaron el total de mapas de la región; estos fueron digitalizados en ArcGIS. Con base en esta fuente, se procedió a georreferenciar y digitalizar nuevamente y, así, obtener una nueva base cartográfica para la investigación en la región. De esta manera, se contó con capas o shapes, una de canales y otra de plataformas, en las que se presenta la información georreferenciada ya registrada, aparte de la importación de nuevas capas que se crearon posteriormente.

Para el modelamiento digital se utilizaron los programas ArcGIS 9.3, Excel 2007 y AutoCAD 2011, los cuales sirvieron para modificar y hacer diversos procesos encaminados a obtener diferentes capas y crear nuevas informaciones que permitieran el manejo y cruce de datos geográficos tabulados y, de ese modo, observarlos en distintos formatos.

También se contó con una capa genérica de los municipios colombianos, en la que se especifica la ubicación espacial, geográfica y administrativa del área, 
que sirvió como base mínima de conocimiento y referencia del proceso. Además, se utilizó una capa de puntos tomados en campo con ayuda de un instrumento receptor de ubicación satelital (GPS). Estos puntos solo se usaron como referencia para identificar el área investigada. Actualmente se está cruzando dicha información y se generarán nuevos mapas (Rojas 2015).

Una vez identificadas las capas que se utilizarían, algunas de las cuales ya existían y otras que fueron creadas, como se describió anteriormente, se inició el proceso de ajuste referencial, procurando que todas las capas tuviesen la misma ubicación espacial, de modo que fuera posible su integración. Posteriormente se georreferenciaron los planos de investigaciones anteriores (Plazas et al. 1993), en los cuales se encuentra registrada información de túmulos funerarios en el área de estudio. Esto se hizo para tener un espectro más amplio de información suministrada por investigaciones anteriores, pero teniendo en cuenta que estas estructuras no se han confirmado en campo. Una vez sumada esta nueva información, se hicieron las correcciones necesarias, especialmente porque era indispensable georreferenciar el plano tomado, ya que este carece de coordenadas en su versión de texto, por lo que se utilizó la corrección de puntos relacionando las entidades dibujadas en el formato análogo y las presentes en el archivo shape de polígonos de plataformas. Después de hacer esto, fue posible ubicar los puntos de los túmulos funerarios de la planimetría existente, y se diseñó un nuevo shape tipo punto y una nueva columna en el cuadro de atributos en el shape de plataformas, donde se consignaron los nuevos elementos.

Finalmente, con todos los elementos estandarizados, de modo que fuera posible su integración y uso como instrumentos de consulta, se procedió a dar respuesta a las siguientes preguntas, teniendo en cuenta de nuevo que dicha información corresponde al último momento de ocupación de la región, lo que no significa que todas las plataformas se hayan usado de forma simultánea, ni siquiera en ese instante: ¿Cuántas plataformas existen en el área aledaña al caño Rabón y cuántas en el caso particular del sitio arqueológico de San Pedro? ¿Cuál es el tamaño promedio de las plataformas de este sector de la Depresión Momposina? ¿Qué características espaciales tiene la concentración de canales y camellones en el sitio de San Pedro? ¿Existe relación entre las plataformas y los campos de cultivo en cuanto a formas y tamaños? Estos interrogantes se agruparon alrededor de los siguientes objetivos específicos: en primer lugar, determinar el tamaño de las plataformas y, en segundo lugar, identificar los centros y dispersiones de las plataformas en la región de estudio. 
Figura 3. Plataforma del poblado de San Pedro

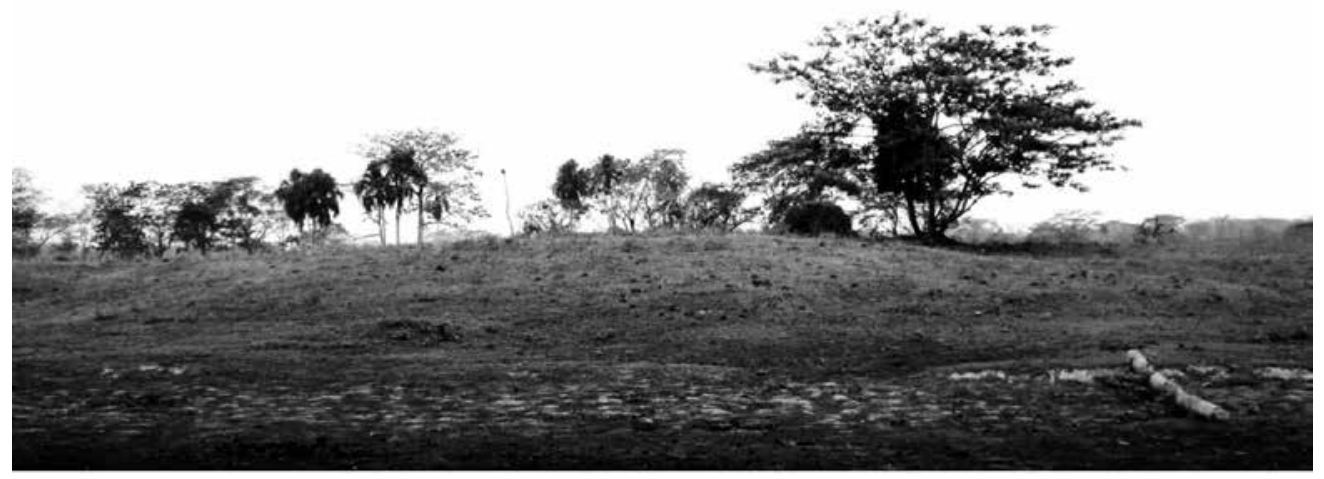

Fuente: Fotografía de Sneider Rojas Mora.

Figura 4. Canales y camellones que circundan el poblado de San Pedro

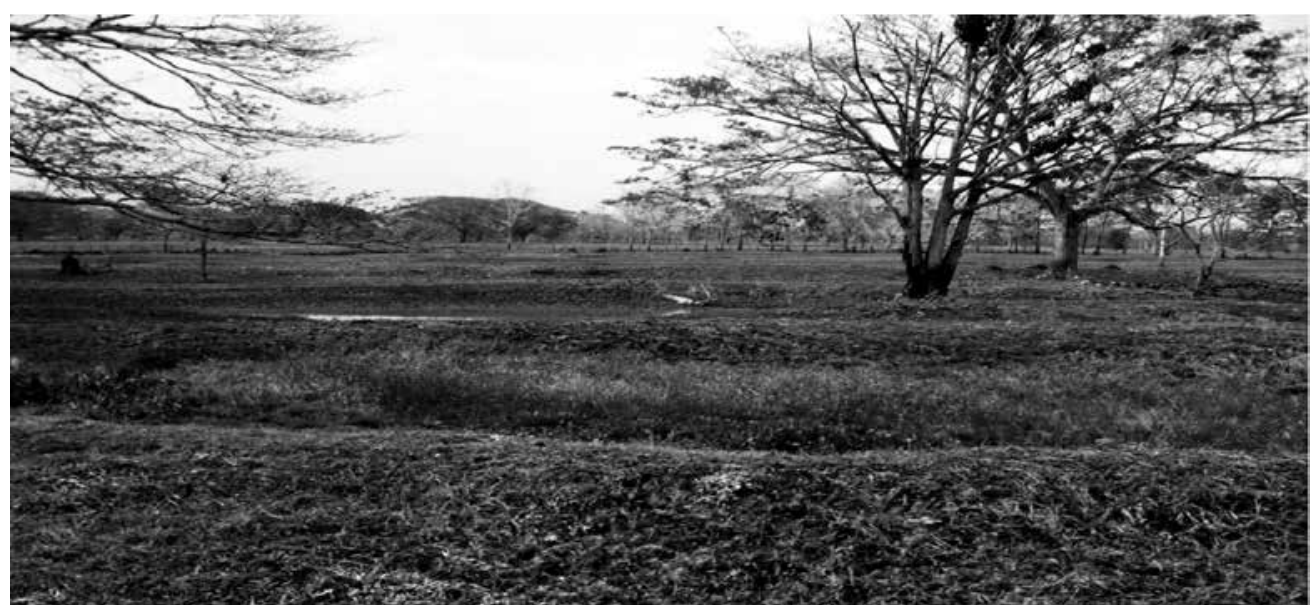

Fuente: Fotografía de Sneider Rojas Mora.

\section{Resultados}

La zona de estudio, que comprende un área total de aproximadamente $49 \mathrm{~km}^{2}$, se dividió en: 1) poblado central (figura 3); 2) zona de canales y camellones que circundan el lugar central (figura 4), y 3) zona externa a los camellones con presencia de plataformas. A efectos del análisis, se consideraron la zona central y la zona externa a los camellones y con presencia de plataformas. Este artículo 
expone los resultados del análisis espacial de estas áreas y el estudio de la zona de canales y camellones que circundan el lugar central y que es objeto de otra investigación (Rojas 2015). En la primera zona, el lugar central de San Pedro, se reportaron 53 plataformas con perímetro promedio de 119,71 metros, de las cuales las más pequeñas fueron aquellas con perímetro de 101,13 metros y las más grandes, aquellas que tenían 154,94 metros. Entre tanto, en la zona externa a los camellones se contaron 742 plataformas, con un promedio de perímetro de 115,7 metros, de las cuales las más pequeñas fueron aquellas cuyo perímetro estuvo alrededor de 100,11 metros y las más grandes, aquellas con perímetro equivalente a 187,38 metros.

Lo anterior significa que no existe una gran diferencia en el tamaño promedio de las plataformas de un sector y otro, a pesar de que las de mayor tamaño se encuentran en el sector externo a San Pedro. En vista de esta observación, se decidió hacer una relación de la cantidad de plataformas que, tanto en uno como en otro sector, tenían los tamaños más significativos, con lo cual se logró una diferenciación entre los tamaños y el número de plataformas que estaban en dichos rangos. Estos se establecieron de manera arbitraria, pues no se cuenta con excavaciones en área de plataformas en la región de estudio, excepción hecha de unos pozos de control estratigráficos y una trinchera en una plataforma del sitio La Bastilla (Rojas y Montejo 2002), y una exploración sistemática mediante sondeos y trincheras en el sitio San Pedro, cuyo material arqueológico se está estudiando (Rojas 2015). Los resultados de esta observación del área de influencia del caño Rabón se expresan en la tabla 1. Por su parte, los resultados de la observación en el sitio San Pedro se presentan en la tabla 2.

Tabla 1. Distribución de plataformas por rango de tamaño. Área de influencia del caño Rabón

\begin{tabular}{c|c}
\hline Rangos en metros de perimetro & Cantidad de plataformas \\
\hline $100-110$ & 276 \\
\hline $110-120$ & 253 \\
\hline $120-130$ & 141 \\
\hline $130-140$ & 39 \\
\hline $140-150$ & 21 \\
\hline $150-160$ & 8 \\
\hline $160-170$ & 3 \\
\hline $170-180$ & 0 \\
\hline $180-190$ & 1 \\
\hline
\end{tabular}

Fuente: Elaboración de Joyce Pertuz. 
Tabla 2. Distribución de plataformas por rango de tamaño. Área de influencia del sitio San Pedro o poblado central

\begin{tabular}{c|c}
\hline Rangos en metros de perímetro & Cantidad de plataformas \\
\hline $100-110$ & 12 \\
\hline $111-120$ & 25 \\
\hline $121-130$ & 7 \\
\hline $131-140$ & 6 \\
\hline $141-150$ & 3 \\
\hline $151-160$ & 1 \\
\hline
\end{tabular}

Fuente: Elaboración de Joyce Pertuz.

Es evidente que en ambos casos, del total de plataformas, el 37,2\% tiene un tamaño perimetral de entre 100 metros y 110 metros, el 34,1\% está entre 110 metros y 120 metros, mientras que el 1\% lo constituyen aquellas de dimensiones mayores a 160 metros y hasta 190 metros, aunque no se registran elementos con medidas de entre 170 metros y 180 metros. En cuanto a las plataformas del sector de San Pedro, el 24,53\% se ubica en el rango de 111 metros a 115 metros de perímetro, el 22,64\% está entre 115 metros y 120 metros y el 1,8\%, en el rango de mayor tamaño: de 141 metros a 145 metros y de 150 metros a 155 metros.

Una vez que se hizo este ejercicio, se continuó con la creación de planos o mapas de densidades, se verificó su georreferencia y se sumaron los tamaños de las capas. De esta manera, se procura evitar incompatibilidades y planos de respuesta errados. Para este paso, no se separaron las áreas aledañas al caño Rabón de aquellas presentes en el poblado central de San Pedro, como en el proceso anterior, sino que se tomó como un sector completo e integrado. Luego se georreferenciaron el plano y los mapas diseñados utilizando las herramientas de georreferencia y adición de puntos de control (georeferencing y add control points), hasta que su ubicación coincidiera con la capa de base shape de plataformas. Luego se creó una nueva capa de tipo punto, nombrada como túmulos funerarios, donde se ubicaron los puntos copiados del plano físico.

El siguiente paso consistió en generar los planos de distancia y dispersión de toda la zona de estudio. Para el primer plano se utilizó la capa shape de plataformas y se aplicó el buffer de distancia con diferentes valores que no generaron un plano óptimo de consulta, ya que no se interrelacionaban los elementos sino que se los tomaba independientemente. Luego se revisó en la caja de herramientas (toolbox), en las herramientas de análisis espacial (spatial analyst) y en las de distancia (distance), y se utilizó distancia en línea recta entre las entidades. El 
Figura 5. Distribución de plataformas concentradas en área central y dispersión en zona externa. Túmulos funerarios

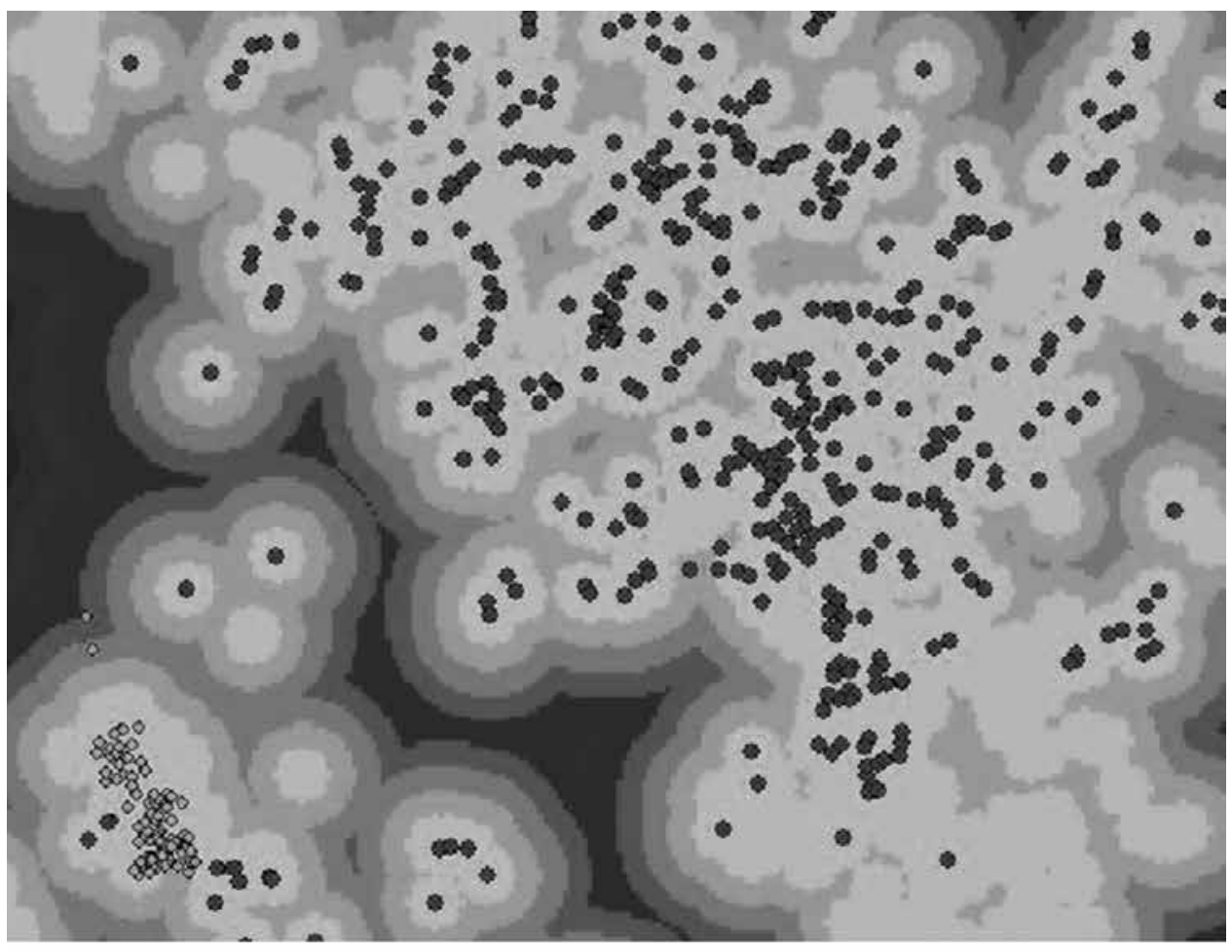

$\begin{array}{lrrr}1600 \quad 800 \quad 0 & 1600 \text { metros }\end{array}$

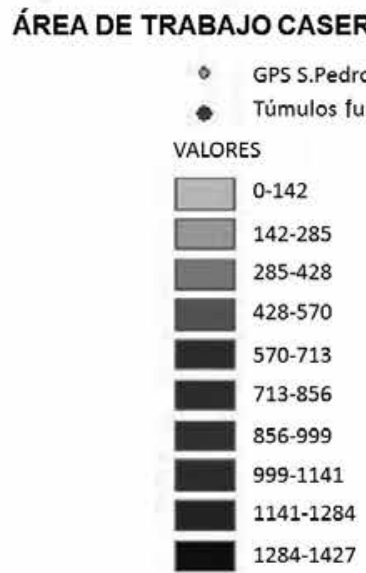

Fuente: Elaboración propia. 
resultado fue un mapa con una clasificación de 10 valores que van desde los 0 metros hasta los 142 metros (figura 5).

Posteriormente se generaron conjuntos de conglomerados denominados cluster analysis, en los que se agruparon las plataformas según la distancia que había entre ellas. De este ejercicio se obtuvo un total de 34 clústers, que variaron en número de plataformas: los de menor número fueron aquellos que agruparon 3 elementos, mientras que hubo otro en el que se registraron 58 plataformas, aunque es evidente que este clúster no es el que corresponde al denominado poblado central, que, como hemos dicho en otras oportunidades, cuenta con 53 plataformas. El tamaño promedio del perímetro de las plataformas fue de 115 metros; fue llamativo el clúster marcado con el número 27, ubicado en el costado occidental del área de estudio y cuyo perímetro promedio fue de 150,19 metros. En la tabla 3 se detallan los resultados del análisis espacial, que incluyó conjuntos o clústeres, número de plataformas, tamaños mínimos y máximos y su promedio.

Tabla 3. Número de clústeres, densidades y perímetros de plataformas

\begin{tabular}{|c|c|c|c|c|}
\hline \multicolumn{5}{|c|}{ Clúster de plataformas. Rangos de densidad } \\
\hline Nombre - clúster & N. ${ }^{\circ}$ entidades & $\begin{array}{l}\text { Tam. mín. en } \\
\text { metros }\end{array}$ & $\begin{array}{l}\text { Tam. máx. } \\
\text { en metros }\end{array}$ & $\begin{array}{l}\text { Prom. tam. } \\
\text { en metros }\end{array}$ \\
\hline 1 & 53 & 101,13 & 154,95 & 119,71 \\
\hline 2 & 56 & 102,35 & 146,82 & 118,19 \\
\hline 3 & 58 & 100,55 & 127,9 & 110,92 \\
\hline 4 & 23 & 102,86 & 126,17 & 112 \\
\hline 5 & 15 & 106,7 & 135,36 & 120,08 \\
\hline 6 & 18 & 101,72 & 128,33 & 116,9 \\
\hline 7 & 20 & 103,62 & 140,83 & 122,39 \\
\hline 8 & 7 & 102,45 & 127,99 & 112,5 \\
\hline 9 & 9 & 101 & 125,65 & 112,19 \\
\hline 10 & 12 & 103,16 & 137,32 & 113,53 \\
\hline 11 & 9 & 101,54 & 127,95 & 112,39 \\
\hline 12 & 7 & 100,42 & 135,84 & 116,91 \\
\hline 13 & 11 & 102,74 & 124,87 & 112 \\
\hline 14 & 13 & 100,77 & 112,9 & 105,1 \\
\hline 15 & 15 & 100,82 & 149,17 & 117,45 \\
\hline 16 & 15 & 100,81 & 116,06 & 107,76 \\
\hline 17 & 15 & 107,36 & 139,61 & 120,43 \\
\hline 18 & 5 & 115,35 & 137,98 & 124,62 \\
\hline 19 & 7 & 128,71 & 154,48 & 142,47 \\
\hline
\end{tabular}


Clúster de plataformas. Rangos de densidad

\begin{tabular}{c|c|c|c|c}
\hline 20 & 4 & 129,57 & 164,06 & 140,23 \\
\hline 21 & 9 & 107,75 & 132,7 & 116,88 \\
\hline 22 & 6 & 100,15 & 118,9 & 106,4 \\
\hline 23 & 5 & 106,22 & 121,1 & 112,19 \\
\hline 24 & 7 & 100,3 & 121,42 & 111,64 \\
\hline 25 & 6 & 110,29 & 141,02 & 123,89 \\
\hline 26 & 3 & 127,71 & 146,7 & 138,44 \\
\hline 27 & 3 & 133,89 & 164,05 & 150,19 \\
\hline 28 & 3 & 114,94 & 130,29 & 121,95 \\
\hline 29 & 12 & 105,08 & 134,33 & 119,42 \\
\hline 30 & 9 & 100,49 & 122 & 111,11 \\
\hline 31 & 16 & 104,66 & 125,91 & 115,38 \\
\hline 32 & 9 & 100,48 & 131,88 & 109,12 \\
\hline 33 & 6 & 105,54 & 122,08 & 113,31 \\
\hline 34 & 8 & 102,91 & 129,04 & 116,29 \\
\hline
\end{tabular}

Fuente: Elaboración de Joyce Pertuz.

Con los resultados cuantitativos de esta tabla, se diseñó una imagen (figura 6) en la que se pueden observar claramente las relaciones antes señaladas, así como la ubicación de los puntos GPS5 tomados en campo y las distancias entre las plataformas. Además, se muestra la ubicación de los túmulos funerarios.

En el segundo plano, denominado de dispersión, se utilizó nuevamente la capa shape de plataformas y se aplicó la herramienta análisis espacial (spatial analyst), pero esta vez se utilizó densidad (density). El resultado es un plano que muestra la acumulación o separación de las plataformas en este espacio, lo que permite ver los lugares donde se ubican los clústeres de elementos, concentrados principalmente en 10 sitios. El mapa generó una escala de colores desde el amarillo, que corresponde a aquellas áreas con nula cantidad de elementos, hasta el azul oscuro, que indica la mayor concentración, correspondiente a un promedio de 19,86 plataformas por kilómetro cuadrado. A este plano se superpuso la capa shape de túmulos funerarios dibujados.

En la figura 7 se observa la concentración de plataformas y la ubicación de los túmulos funerarios. En ella se muestran la ubicación de los puntos GPS5 tomados en campo y las distancias entre las plataformas.

La investigación llevada a cabo en la región de San Pedro, municipio de San Benito Abad, fue el resultado de un trabajo de campo cuyos tiempos se vieron comprometidos, debido a la inundación que sufrió la región por causa del fenómeno de la Niña que se vivió en el país entre mayo de 2011 y 2012. Esta situación hizo que el trabajo se concentrara en la investigación preliminar, y que 
Figura 6. Concentración de plataformas en el área de estudio

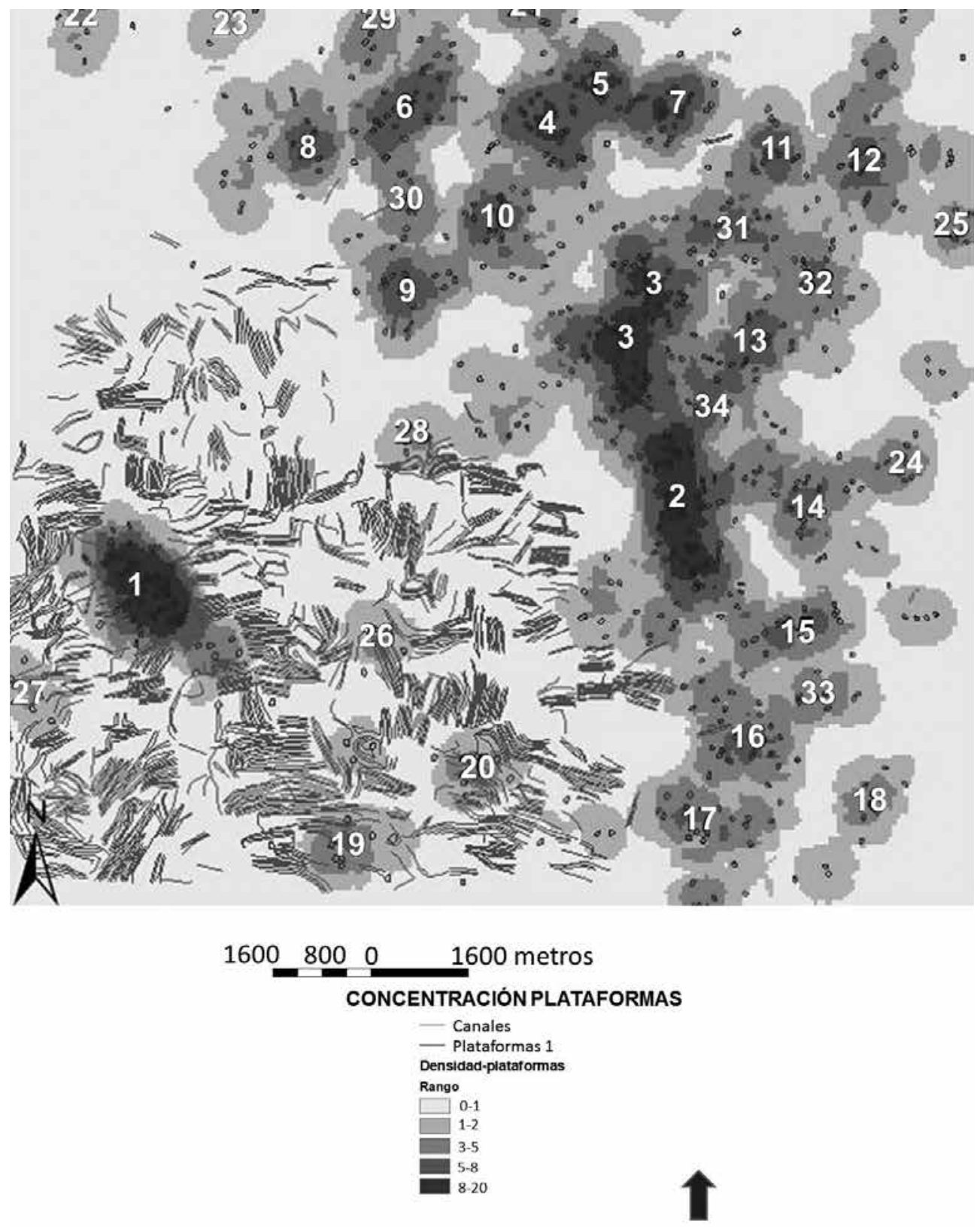

Fuente: Elaboración propia. 
Figura 7. Concentración de plataformas y ubicación de los túmulos funerarios

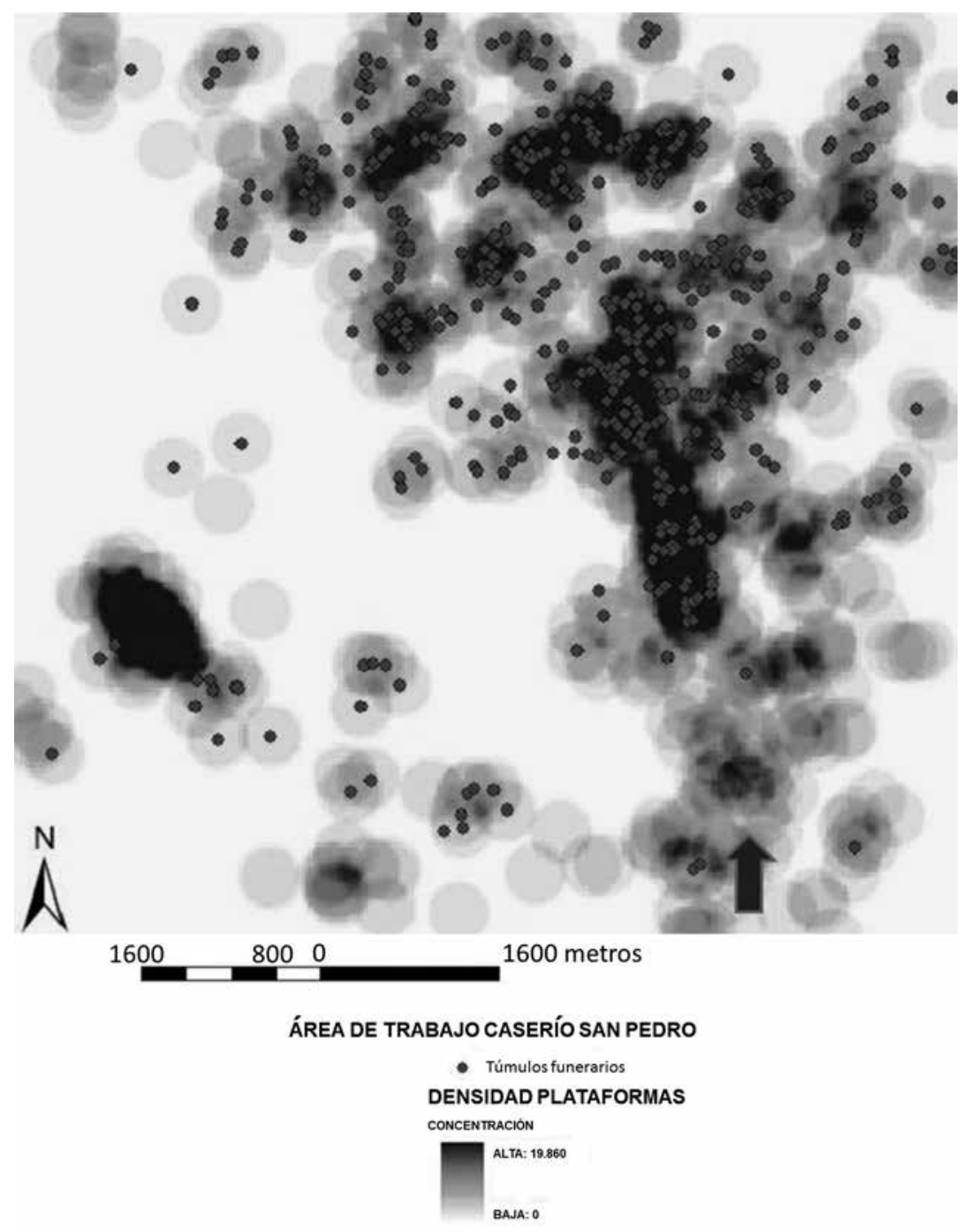

Fuente: Elaboración propia.

el campo y el laboratorio se vieran limitados a tres meses. Esto redundó en la imposibilidad de contar con los análisis palinológicos y de suelos, a pesar de que se tomaron muestras para ellos.

No obstante lo anterior, consideramos que los resultados de la presente investigación constituyen un aporte importante al conocimiento de la región de estudio y abren un camino para las investigaciones que se ocupan de estudiar 
las sociedades complejas en la Depresión Momposina, particularmente en tradiciones de temprana ocupación de la región.

\section{Conclusiones}

El sector de San Pedro es uno de los que cuenta con mayor número de canales y camellones entrecruzados en la Depresión Momposina, con una concentración de plataformas tanto en el centro como en las áreas externas y de influencia del caño Rabón. En esta zona, Plazas et al. (1993) reportaron la existencia de plataformas con elevaciones, lo que les hizo pensar que se trataba de túmulos funerarios. Esta situación no se ha confirmado en campo y solo se usó en este trabajo como referencia para anclar plataformas y facilitar su georreferenciación.

De igual forma, de la presente investigación surgieron dos consideraciones: la primera, obtenida a partir del análisis de ubicación y distribución espacial de las plataformas en la región de estudio, es que en la zona hay varios conjuntos que pudieron haber fungido como poblados de un lugar ubicado en el área denominada en esta investigación zona central. Esta, si bien no tiene túmulos funerarios en su interior, debió de ser importante en la administración de los recursos, producto del aprovechamiento de los camellones aledaños, los cuales fueron objeto de la investigación llevada a cabo por Aristizábal (2012). No obstante, surgen varios interrogantes, que solo podrán ser resueltos con trabajo de campo posterior: ¿Corresponden estas unidades espaciales a un mismo momento histórico? Asumiendo que fuera así, si la zona denominada poblado central corresponde a un lugar central, ¿qué relación tendría con las áreas aledañas? ¿Qué marcadores de centralidad se pueden encontrar? ¿Existe alguna relación entre las concentraciones externas, incluso más grandes, y las concentraciones del poblado de San Pedro? ¿Son contemporáneas dichas concentraciones? Y un hecho que llama la atención, por la distribución, es ¿por qué el poblado de San Pedro está encerrado en medio de grandes extensiones de canales y camellones?

Una segunda consideración es que la zona parece haber sido ocupada únicamente alrededor del siglo VII d. C., como lo confirman las fechas obtenidas en la zona (Plazas et al. 1993; Rojas 2015).

Ahora, teniendo en cuenta que aún hacen falta otros resultados de investigación, entre los que se cuentan fundamentalmente los estudios cronológicos de cerámica y la datación radiocarbónica en plataformas y camellones, así como la confirmación en campo de la presencia de túmulos funerarios, los resultados acá expuestos nos permiten reflexionar acerca de temáticas relacionadas con la 
región que no se han abordado de manera sistemática. Por ejemplo, la cuestión de si existe una correspondencia positiva entre los lugares donde se concentró la población y el control político. Tampoco se ha reflexionado respecto al desarrollo de jerarquías de asentamiento, reflexión que en otras regiones ha permitido considerar el tamaño de los sitios. De igual forma, estas consideraciones conducen a pensar en la necesidad de estudiar la manera en que la agrupación espacial se relaciona con la disminución de costos de transporte, lo que favorece una mejor circulación de información y, por consiguiente, un mayor control (Montmollin 1987). En este sentido, una ubicación alejada de los lugares centrales se concibe como una táctica para reducir el control de los ejes centrales.

Finalmente, resta decir que el presente documento constituye una posibilidad de acercarse al análisis espacial de la región y, a partir de ello, a la consideración de interrogantes sobre el tipo de sociedades que llevaron a cabo las modificaciones mencionadas.

\section{Agradecimientos}

Los autores desean expresar sus más sinceros agradecimientos a los campesinos y pescadores de la región; al señor Filadelfo Monterroso y su familia, propietarios de la hacienda Tuloviste, en donde se encuentra la "mayoría" de San Pedro. A don José y doña Carmen, administradores de la hacienda. A los trabajadores de campo Eliécer y Wílmar. A la antropóloga de la Universidad de Antioquia Joyce Pertuz, quien hizo los análisis espaciales, base de este documento. Igualmente, al entonces estudiante (2012) y hoy graduado de Antropología de la Universidad de Antioquia, señor Mateo Aristizábal, quien acompañó la temporada de campo y comprendió los rigores que implica el estudio de esta región. 


\section{Referencias}

Agujera Díaz, María. 2005. La economía del departamento de Sucre: ganadería y sector público. Documentos de Trabajo sobre Economía Regional 63. Cartagena: Banco de la República.

Aristizábal, Mateo. 2012. "San Pedro: del presente al pasado. Una aproximación a la dinámica espacial de las sociedades prehispánicas de la Depresión Momposina (Caribe colombiano)". Tesis de pregrado en Antropología, Universidad de Antioquia, Medellín.

Ballesteros, C. I. 1983. Mapa geológico generalizado del departamento de Bolívar escala: 1:500.000. Bogotá: Ingeominas.

Denevan, William M. 1992. "The Pristine Myth: The Landscapes of the Americas in 1492". Association of American Geographers 82 (3): 369-385.

-. 2001. Cultivated Landscapes of Native Amazonia and the Andes. Nueva York: Oxford University Press.

Denevan, William y Victor Turner. 1974. "Forms, Functions, and Associations of Raised Fields in the Old World Tropics". Journal of Tropical Geography (39): 24-39.

Erickson, Clark L. 2006. "The Domesticated Landscapes of the Bolivian Amazon”. En Time and Complexity in Historical Ecology: Studies in the Neotropical Lowlands, editado por William Balée y Clark L. Erickson, 235-278. Nueva York: Columbia University Press.

-. 2008. "Amazonia: The Historical Ecology of a Domesticated Landscape". En Handbook of South American Archaeology, edited by Helaine Silverman y William Isbell, 157-183. Urbana: University of Illinois at Urbana-Champaign.

-. 2010. "The Transformation of Environment into Landscape: The Historical
Ecology of Monumental Earthwork Construction in the Bolivian Amazon”. Diversity 2: 618-652.

Forero, G., P. Ferreira y M. Maya. 1997. Atlas geológico digital de Colombia (versión 1.0) Plancha 2, escala 1:500.000. Bogotá: Ingeominas.

Hammen, Thomas van der. 1986. "Fluctuaciones holocénicas del nivel de inundaciones en la cuenca del bajo Magdalena-Cauca-San Jorge (Colombia)”. Geología Andina 10: 11-18.

\section{Herrera, Luisa Fernanda y Juan Carlos} Berrío. 1996. "Vegetación natural y acción antrópica durante los últimos 1.000 años: análisis palinológico de un perfil asociado a canales artificiales de San Marcos, Sucre”. Licania Arborea 1 (1): 50-59.

Herrera, Luisa Fernanda, Sneider Rojas y Fernando Montejo. 2004. "Poblamiento prehispánico de la Depresión Momposina: un sistema integrado de manejo sostenible de los ecosistemas inundables”. En Saberes de vida: por el bienestar de las nuevas generaciones, compilado por Roberto Restrepo, 148-173. Bogotá: Siglo del Hombre.

Instituto de Hidrología, Meteorología y Adecuación de Tierras (Himat). 1977a. Geología y geomorfología. Informe final. Proyecto Colombo-Holandés, vol. 1.

-. 1977b. Proyecto cuenca MagdalenaCauca. Informe final. Proyecto ColomboHolandés, vol. 12.

Instituto Geográfico Agustín Codazzi (IGAC). 1986. Estudio general de suelos de los municipios de Caimito, la Unión de Sucre, Majagual, San Benito Abad, San Marcos y Sucre (departamento de Sucre). Bogotá: IGAC.

Instituto Nacional de Vías (Invías). 1999. "Depresión Momposina con proyectos viales considerados para EAR”. <http:// 
wiki.neotropicos.org/index.php?title =Imagen:DepresionMomposina.png>. Consultado el 8 de abril de 2015.

Iriarte, J. 2003. "Assessing the Feasibility of Identifying Maize through the Analysis of Cross-Shaped Size and ThreeDimensional Morphology of Phytoliths in the Grasslands of Southeastern South America”. Journal of Archaeological Science 30: 1085-1094.

Iriarte, J. et al. 2010. "Late Holocene Neotropical Agricultural Landscapes: Phytolith and Stable Carbon Isotope Analysis of Raised Fields from French Guianan Coastal Savannahs”. Journal of Archaeological Science 37: 2984-2994.

Lombardo, Umberto. 2010. "Raised Fields of Northwester Bolivia: A GIS Based Analysis”. Zeitschrift für Archäologie Außereuropäischer Kulturen 3: 127-149.

Lombardo, Umberto et al. 2011. "Raised Fields in the Bolivian Amazonia: A Prehistoric Green Revolution or a Flood Risk Mitigation Strategy?”. Journal of Archaeological Science 38 (3): 502-512.

Montejo, Fernando y Fernando Ávila. 2007. "Modificaciones antrópicas de ambientes de humedad: perspectiva histórica de un análisis espacial utilizando un sistema de información geográfica”. Tesis de Especialización en Sistema de Información Geográfica, IGAC, Bogotá.

Montejo, Fernando y Sneider Rojas. 2009. "Impacto social y ambiental del manejo hidráulico y uso del agua a lo largo de dos mil años en el Caribe colombiano”. En Riesgos ancestrales en Iberoamérica. Técnicas y organización social del pequeño riesgo, editado por Tomás Martínez Saldaña, 63-68. México: Mundi-Prensa.

Montmollin, Oliver. 1987. "Temporal and Social Scale in Prehispanic Mesoamerica”. En Archaeological Review 6 (1): 51-61.

Parason, J. y W. M. Bowen. 1966. “Ancient
Ridged Fields of the San Jorge River Floodplain, Colombia”. Geographical Review 56: 317-343.

Plazas, Clemencia y Ana María Falchetti. 1981. Asentamientos prehispánicos en el bajo río San Jorge. Bogotá: Fundación de Investigaciones Arqueológicas Nacionales, Banco de la República.

Plazas, Clemencia et al. 1993. La sociedad hidráulica zenú. Estudio arqueológico de 2.000 años de historia en las llanuras del Caribe colombiano. Bogotá: Banco de la República.

Plazas, Clemencia et al. 1988. "Cambios ambientales y desarrollo cultural en el bajo río San Jorge”. Boletín del Museo del Oro 20: 55-88.

Rodrigues, L. et al. 2014. "Pre-Columbian Agriculture in the Bolivian Lowlands: Construction History and Management of Raised Fields in Bermeo”. Catena 132: 126-138.

Rojas Mora, Sneider. 2015. "Proyecto de investigación Agricultura y viviendas prehispánicas en el Caribe Colombiano". Manuscrito inédito. Centro de Investigaciones Sociales y Humanas (CISH), CODI y Vicerrectoría de Investigación de la Universidad de Antioquia.

Rojas, Sneider y Fernando Montejo. 1999. "Manejo agrícola y campos de cultivo prehispánico en el bajo río San Jorge”. Manuscrito inédito. Colciencias; Corpoica; Fundación Erigaie.

—. 2002. "Viviendas prehispánicas en el bajo río San Jorge”. Manuscrito inédito. Fundación de Investigaciones Arqueológicas Nacionales, Banco de la República.

Rostain, S. 2010. "Pre-Columbian Earthworks in Coastal Amazonia”. Diversity 2: 331-352.

Walker, John Hamilton. 1999. "Agricultural Change in the Bolivian Amazon”. 
Tesis de doctorado, University of Pennsylvania, Estados Unidos.

-. 2000. "Raised Field Abandonment in the Upper Amazon”. Culture and Agriculture 22 (2): $27-31$.

-. 2004. Agricultural Change in the Bolivian Amazon - Cambio Agrícola en la
Amazonía Boliviana. Memoirs in Latin American Archaeology 13. Pittsburgh: University of Pittsburgh; Fundación Kenneth Lee.

-. 2011. "Social Implications from Agricultural Taskscapes in the Southwestern Amazon”. Latin American Antiquity 22 (3): 275-295. 